efits and risks of chelation therapy in this patient population are necessary. But like Porter and Garbowski, ${ }^{1}$ the panel was concerned that the evidence from randomized trials of chelation therapy in patients with SCD receiving transfusion therapy may require up to 2 decades to test end points such as death or hepatic cirrhosis.

It is not clear that such randomization would even be considered feasible or ethical. Based on this information, the panel chose to make a moderate strength recommendation to consider chelation therapy in patients with SCD and signs of iron overload.

Dr Klings and colleagues address the serious problem of pulmonary hypertension in patients with SCD. Even though our guidelines differ from those of the American Thoracic Society for screening asymptomatic patients with SCD for pulmonary hypertension, we strongly support their recommendation for the conduct of prospective outcome studies of tricuspid regurgitant jet velocity screening accompanied by evaluation of pharmacological intervention in patients in whom cardiac catheterization confirms a diagnosis of pulmonary arterial hypertension or pulmonary venous hypertension. ${ }^{4}$

We do agree that no evidence exists to demonstrate that the screening of asymptomatic patients changes outcomes. We hope that continued consideration will also be given to quantifying the currently unknown number of asymptomatic individuals with treatable contributory factors as well as further clarification of the benefits and harms of screening to allow risk stratification.

We agree with Klings and colleagues that there is continued underuse of SCD-specific therapies. One of the primary goals of our guideline sponsored by the National Heart, Lung, and Blood Institute was to enhance the availability of hydroxyurea and transfusion therapy for all SCD treatment candidates, in agreement with Klings et al.

Barbara P. Yawn, MD, MSc, MSPH

George Buchanan, MD

Kathryn Hassell, MD

Author Affiliations: Department of Research and Education, Olmsted Medical Center, Rochester, Minnesota (Yawn); University of Texas Southwestern Medical Center, Dallas (Buchanan); University of Colorado, Denver (Hassell).

Corresponding Author: Barbara P. Yawn, MD, MSc, MSPH, Department of Research and Education, Olmsted Medical Center, 210 Ninth St SE, Rochester MN 55904 (byawn@olmmed.org).

Conflict of Interest Disclosures: The authors have completed and submitted the ICMJE Form for Disclosure of Potential Conflicts of Interest. Dr Hassell reported receiving individual payment for serving on advisory boards for Pfizer, ApoPharma, the Foundation for Women and Girls with Blood Disorders, and PFO Research Foundation; receiving consultant fees from AGA Medical Corp; receiving individual and institutional grant support from Terumo, GlycoMimetics, and Emmaus; and serving on the board of directors for Mount Evans Home Health \& Hospice. No other disclosures were reported.

1. Porter J, Garbowski M. Consequences and management of iron overload in sickle cell disease. Hematology Am Soc Hematol Educ Program. 2013;2013:447456.

2. Olivieri NF. Progression of iron overload in sickle cell disease. Semin Hematol. 2001;38(1)(suppl 1):57-62
3. Brown K, Subramony C, May W, et al. Hepatic iron overload in children with sickle cell anemia on chronic transfusion therapy. J Pediatr Hematol Oncol. 2009;31(5):309-312.

4. Klings ES, Machado RF, Barst RJ, et al; American Thoracic Society Ad Hoc Committee on Pulmonary Hypertension of Sickle Cell Disease. An official American Thoracic Society clinical practice guideline: diagnosis, risk stratification, and management of pulmonary hypertension of sickle cell disease. Am J Respir Crit Care Med. 2014:189(6):727-740.

\section{Reanalyses of Trial Results}

To the Editor We agree with Dr Ebrahim and colleagues ${ }^{1}$ that reanalyses of clinical trial data provide an important opportunity to better understand trial results and that more independent reanalyses are needed. However, the estimate of the number of reanalyses published prior to March 9, 2014, reported by Ebrahim et $\mathrm{al}^{1}$ is incorrect, possibly by a substantial margin.

Ebrahim et $\mathrm{al}^{1}$ reported that their MEDLINE search identified 37 reanalyses of data from previously published randomized clinical trials. However, the authors limited their search to manuscripts categorized as a clinical trial in MEDLINE. This is problematic because many reanalyses are given the more general classification of journal article and so are not identified in searches restricted to clinical trial articles.

Examples of this can be found among the reanalyses of the data from the trial of tissue plasminogen activator for ischemic stroke sponsored by the National Institute of Neurological Disorders and Stroke (NINDS). The original analysis was published in $1995 .^{2}$ A recent Cochrane review ${ }^{3}$ identified at least 7 reanalyses of the data from the NINDS trial. Each of these reanalyses meets the criteria defined by Ebrahim et $\mathrm{al}^{1}$; however, none was identified using their search method. Five of these reanalyses are not classified within MEDLINE as clinical trials. The same problem may be true of other reanalyses.

As noted by IBM Quality Manager H. James Harrington, "measurement is the first step that leads to control and eventually improvement."4 Obtaining an accurate measure of the number of reanalyses being published and defining a search method that can consistently generate this number is essential to assess whether new policies (such as those called for by Krumholz and Peterson ${ }^{5}$ ) result in the increase in reanalyses needed to optimize understanding of trial results.

A search unrestricted by publication type should provide a more accurate measure of the number of reanalyses but adds considerably to the workload of the search. Steps to improve the consistency of classification of articles by publication type within MEDLINE may provide a long-term solution to this challenge.

\section{Timothy F. Platts-Mills, MD, MSc} Christopher W. Jones, MD

Author Affiliations: Department of Emergency Medicine, University of North Carolina, Chapel Hill (Platts-Mills); Department of Emergency Medicine, Cooper Medical School of Rowan University, Camden, New Jersey (Jones).

Corresponding Author: Timothy F. Platts-Mills, MD, MSc, Department of Emergency Medicine, University of North Carolina, 170 Manning Dr, Chapel Hill, NC 27599 (tplattsm@med.unc.edu). 
Conflict of Interest Disclosures: The authors have completed and submitted the ICMJE Form for Disclosure of Potential Conflicts of Interest and none were reported.

1. Ebrahim S, Sohani ZN, Montoya $L$, et al. Reanalyses of randomized clinical trial data. JAMA. 2014;312(10):1024-1032.

2. National Institute of Neurological Disorders and Stroke rt-PA Stroke Study Group. Tissue plasminogen activator for acute ischemic stroke. N Engl J Med. 1995;333(24):1581-1587

3. Wardlaw JM, Murray V, Berge E, del Zoppo GJ. Thrombolysis for acute ischaemic stroke. Cochrane Database Syst Rev. 2014;7:CD000213.

4. Kaydos W. Operational Performance Measurement. Boca Raton, FL: CRC Press; 1999.

5. Krumholz HM, Peterson ED. Open access to clinical trials data. JAMA. 2014;312 (10):1002-1003.

In Reply Drs Platts-Mills and Jones have raised a potential concern with the search strategy used in our study on reanalyses of randomized clinical trial data, specifically with respect to our restriction to studies indexed as a clinical trial publication type in MEDLINE. We agree with them that a strategy with no restriction to clinical trials publication type would be more sensitive in detecting reanalyses.

However, performing such a search would yield a total of 112330 citations (limited to March 9, 2014, as per our original search). Screening this number of citations would be a daunting task and may not be a valuable use of resources if the output of additional studies retrieved would be few in number compared with the studies retrieved in our original search.

We have attempted to get an estimate of how many studies we potentially missed by using a simplified search with (reanalysis [ti] OR reanalysis [ti]) NOT Clinical Trial [PT] AND random* (limited to March 9, 2014) that would capture the reanalysis of the NINDS rt-PA Stroke Study. Besides the NINDS study, we also identified eligible reanalyses for 3 more trials. Two of the 4 new eligible trials ${ }^{1-4}$ had their reanalyses completed by independent authors or committees, ${ }^{2,3}$ and the other 2 had reanalyses completed by the same research group or included authors from the original study. ${ }^{1,4}$

Reasons for completing reanalyses included the use of intention-to-treat principles (compared with per-protocol analyses used in the original article), ${ }^{1}$ correction for subgroup imbalances, ${ }^{3}$ use of a method for identifying latent trajectories (latent class growth analysis), ${ }^{4}$ and comparison of the primary end point using a change in the definition of the outcome. $^{2}$

Of the 4 reanalyses, 1 found that the experimental treatment (bilavirudin) was significantly more effective and had less bleeding complications than control (heparin), ${ }^{1}$ whereas the original analysis had shown no significant difference in efficacy, so perhaps this would lead to more patients being treated with bilavirudin. Another reanalysis ${ }^{4}$ found effects for latent classes of participants that were not obvious in the original analysis, so it is possible that different patients may be treated differently based on these results.

We have no doubt that a few more trials must have been missed given the imperfect sensitivity of any searches and the lack of a standard tag for reanalyses in PubMed. However, we believe that it is unlikely that the number of missed published reanalyses is large. Measures to improve awareness of the importance and systematic indexing of reanalyses in PubMed are certainly required.

Nonetheless, our main conclusions that published reanalyses are currently rare, almost always involve authors of the original analyses, and about one-third reach different conclusions still hold true. If there were more interest in publishing reanalyses regardless of their results, we suspect that the proportion of those claiming that they had found something different from the original analysis would decrease.

Shanil Ebrahim, PhD

John P. A. Ioannidis, MD, DSc

Author Affiliations: Meta-Research Innovation Center at Stanford, Stanford University, Stanford, California.

Corresponding Author: John P. A. Ioannidis, MD, DSc, Meta-Research Innovation Center at Stanford, Stanford University, 1265 Welch Rd, Stanford, CA 94305 (jioannid@stanford.edu).

Conflict of Interest Disclosures: The authors have completed and submitted the ICMJE Form for Disclosure of Potential Conflicts of Interest and none were reported.

1. Bittl JA, Chaitman BR, Feit F, Kimball W, Topol EJ. Bivalirudin versus heparin during coronary angioplasty for unstable or postinfarction angina: final report reanalysis of the Bivalirudin Angioplasty Study. Am Heart J. 2001;142(6):952-959.

2. Moses RG. Achieving glycosylated hemoglobin targets using the combination of repaglinide and metformin in type 2 diabetes: a reanalysis of earlier data in terms of current targets. Clin Ther. 2008;30(3):552-554.

3. Ingall TJ, O'Fallon WM, Asplund K, et al. Findings from the reanalysis of the NINDS tissue plasminogen activator for acute ischemic stroke treatment trial. Stroke. 2004;35(10):2418-2424.

4. Lennon MC, McAllister W, Kuang L, Herman DB. Capturing intervention effects over time: reanalysis of a critical time intervention for homeless mentally ill men. Am J Public Health. 2005;95(10):1760-1766.

\section{Risk Profile of Clinical Trial Participants}

To the Editor In their research letter, Dr Udell and colleagues ${ }^{1}$ stated that clinical trial participants had a lower risk profile and a more favorable prognosis compared with the broader population included in the National Cardiovascular Data Registry (NCDR) Acute Coronary Treatment and Intervention Outcomes Network Registry-Get With The Guidelines.

It is likely that these differences would be more pronounced if clinical trial participants were compared with patients with myocardial infarction in the broader US population because participation in the NCDR is voluntary and includes participation by only $5 \%$ of US hospitals. ${ }^{1,2}$ In addition, the data would be put in better perspective if the age of the patients with myocardial infarction in the NCDR and the rates of diagnostic cardiac catheterization and percutaneous coronary intervention were compared with a more inclusive database such as the Nationwide Inpatient Sample. ${ }^{2}$

It would also be interesting to compare the characteristics of patients presenting to the NCDR hospitals that did and did not enroll patients in clinical trials because there may be relevant differences in clinical presentation and outcomes. In addition, the better outcomes of participants in clinical trials may be expected from the exclusion criteria of the clinical trial protocols, self-selection of clinical trial participants (who of- 